\title{
Neuer Verbundmaster: Angewandte Gerontologie
}

\section{Erste wissenschaftliche Weiterbildungen in der Gerontologie}

") Die Katholische Hochschule Freiburg, die Hochschule Mannheim und die Katholische Stiftungsfachhochschule München bieten ein neues und einmaliges Verbundprojekt in der $\mathrm{Ge}$ rontologie an. Sie entwickeln derzeit den Verbundmaster Zukunft Alter: Angewandte Gerontologie. An den drei beteiligten Hochschulen können jeweils losgelöst voneinander, berufsbegleitend und praxisnah Wissenschaftliche Weiterbildungen auf Master-Niveau absolviert werden.

Die Wissenschaftlichen Weiterbildungen sind gleichermaßen theorieund anwendungsorientiert. Vermittelt werden Forschungsergebnisse relevanter Wissenschaftsbereiche, die sich mit Fragen des Alterns befassen. Gewonnenes Wissen kann unmittelbar im Berufsalltag angewandt werden. In den festen Gruppen mit maximal 20 Teilnehmenden sind noch wenige Plät- ze frei. Interessierte haben die Wahl zwischen drei Wissenschaftlichen Weiterbildungen:

Multidisziplinäre Interventionsgerontologie und Gerontopsychiatrie Altern in Sozialraum und Quartier Kommunale Beratung und Vernetzung Gesundheit, Case Management und Planung

Das Projekt wird im Rahmen der Förderlinie „Auf- und Ausbau von Strukturen der wissenschaftlichen Weiterbildung an Hochschulen in Baden-Württemberg" aus Mitteln des Europäischen Sozialfonds (ESF) sowie aus Mitteln des Landes Baden-Württemberg finanziert.

www.zukunft-gerontologie.de

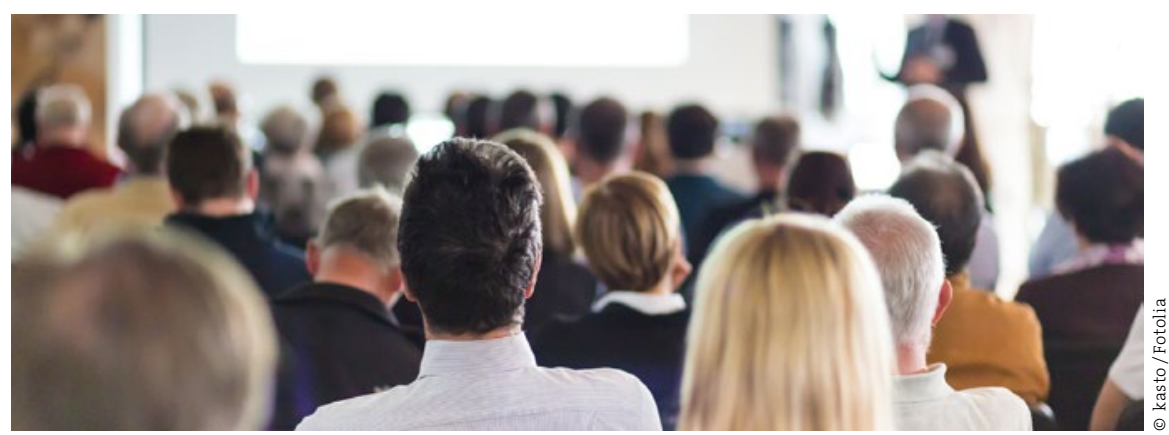

\section{Nachwuchsförderung in NRW}

\section{hsg Bochum unterstützt das Graduierteninstitut}

» Anfang 2016 war das Graduierteninstitut für angewandte Forschung der Fachhochschulen in Nordrhein-Westfalen (GI NRW) gegründet worden. Mittlerweile arbeiten sieben Wissenschaftler der Hochschule für Gesundheit (hsg) in den Fachgruppen des Graduierteninstituts mit. Das GI NRW hat als gemeinsame wissenschaftliche Einrichtung der NRW-Fachhochschulen den Auftrag, kooperative Promotionen an den Hochschulen und Universitäten nachhaltig zu stärken und auszubauen. Interessierte und qualifizierte Professoren der Hochschulen und Universitäten sollen im GI NRW vernetzt werden und gemeinsam Doktoranden betreuen.

Den Kern des Graduierteninstituts bilden die Fachgruppen, in denen sich Professoren mit Promovierenden vernetzen, um sich fachlich auszutauschen und Forschungs- und Promotionsvorhaben $\mathrm{zu}$ initiieren. Als erste Fachgruppe hatte sich im Juli 2016 die Fachgruppe „Soziales und Gesundheit“ gegründet, die die Bereiche Soziales, Gesundheit und Pflege umfasst. Die Fachgruppe bietet forschungsinteressierten Professoren eine Plattform, um dort zusammenzuarbeiten, den Forschungsdiskurs zu beleben und vor allem den Nachwuchs durch kooperative Promotionsverfahren zu fördern. Aktuell arbeiten 47 Mitglieder aus Fachhochschulen beziehungsweise Hochschulen für angewandte Wissenschaften in der Fachgruppe zusammen. Außerdem arbeiten derzeit 30 Kooperationspartnern aus Universitäten und fünf kooperativ promovierende Mitglieder mit.

„Ich freue mich sehr, dass ich im Juli 2017 in die Fachgruppe ,Soziales und Gesundheit' des GI NRW aufgenommen wurde", sagte Prof. Dr. Nicola Bauer, Leiterin des Studienbereichs Hebammenwissenschaft an der Hochschule für Gesundheit (hsg) in Bochum, und führt weiter aus: „Aktuell haben wir in unserem Forschungsprojekt ,HebAB.NRW - Geburtshilfliche Versor- gung durch Hebammen in NordrheinWestfalen' zwei wissenschaftliche Mitarbeiterinnen, die hier promovieren möchten. Auch sie sind Mitglied im Graduierteninstitut.“ „Für uns wissenschaftliche Mitarbeiterinnen bietet das GI NRW, die Möglichkeit uns mit anderen wissenschaftlichen Mitarbeitern aus verschiedenen Fachbereichen an Fachhochschulen auszutauschen, die ebenfalls im Prozess der Promotion sind und so Erfahrungen zu sammeln und Netzwerke zu knüpfen. Zudem bieten die Newsletter des GI NRW zahlreiche Hinweise auf hilfreiche Veranstaltungen und Projekte, wie zum Beispiel eine Schreib-Challenge für Promovierende. Das GI NRW kann es auch erleichtern, einen Betreuenden für eine kooperative Promotion zu finden", fasst Mirjam Peters die Vorteile der Mitgliedschaft zusammen.

www.hs-gesundheit.de 